\title{
Spectral properties and magneto-optical excitations in semiconductor double-rings under Rashba spin-orbit interaction
}

\author{
Wen-Hsuan Kuan, ${ }^{1}$ Chi-Shung Tang, ${ }^{2}$ and Cheng-Hung Chang ${ }^{1,3}$ \\ 1 Physics Division, National Center for Theoretical Sciences, Hsinchu 30013, Taiwan, R.O.C. \\ ${ }^{2}$ Research Center for Applied Sciences, Academia Sinica, Taipei 11529, Taiwan, R.O.C. \\ 3 Institute of Phyics, National Chiao Tung University, Hsinchu 30013, Taiwan, R.O.C.
}

\begin{abstract}
We have numerically solved the Hamiltonian of an electron in a semiconductor double ring subjected to the magnetic flux and Rashba spin-orbit interaction. It is found that the Aharonov-Bohm energy spectrum reveals multi-zigzag periodic structures. The investigations of spin-dependent electron dynamics via Rabi oscillations in two-level and three-level systems demonstrate the possibility of manipulating quantum states. Our results show that the optimal control of photon-assisted inter-ring transitions can be achieved by employing cascade-type and $\Lambda$-type transition mechanisms. Under chirped pulse impulsions, a robust and complete transfer of an electron to the final state is shown to coincide with the estimation of the Landau-Zener formula.
\end{abstract}

PACS numbers: $74.25 . \mathrm{Gz}, 71.70 . \mathrm{Ej}, 74.25 . \mathrm{Nf}$

\section{INTRODUCTION}

The progress in epitaxial growth promotes the use of low-dimensional semiconductor nanostructures in optoelectronic devices. Investigations on fundamental physical properties such as the electronic structure and the carrier population can be directly measured and estimated from the photoluminescence spectrum. Theoretically, several proposals on magneto-optical studies for quantum dot systems have been put forward in the last

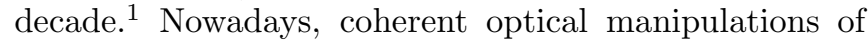
single quantum systems have attracted further attention. The mature technologies in optical control and measurements provide the great opportunity to realize the quantum qubits as logical gates 2 on storage and quantum information processing. ${ }^{\underline{3}}$

In 1990s, the progress of technology has enabled the experimental study of a mesoscopic ring threaded by a static magnetic field displays persistent currents,, .5 which oscillate as a function of magnetic flux $\Phi$ with a period $\Phi_{0}=h c / e$. Recently, applications on spin-orbit interaction (SOI) originated from the breaking of inversion symmetry that gives rise to intrinsic spin splitting in semiconductor systems open a field of spintronics. It was pointed out that the quantum transport of electrons in a spin-polarized system differs greatly from that in a spindegenerate device. $\underline{\underline{6}}$ The utilization of the spin degree of freedom offers the mechanism to speed up quantum information processing. In nature electronic devices such as the Datta-Das transistor, $\stackrel{7}{=}$ spin-waveguide ${ }^{\underline{8}}$ and spinfilter ${ }^{9}$ were proposed.

Several theoretical works associated with Rashba SOI due to structural inversion asymmetry in quantum dot systems were studied $\stackrel{10}{*}$ More recently, the success on self-assembled formation of concentric quantum double rings $\frac{11}{1}$ provides a new system to explore electron dynamics by magneto-optical excitations on the basis of fully analyzed signature of $\mathrm{AB}$ spectrum within the effect of Rashba SOI. The radius of flat double rings is about
$100 \mathrm{~nm}$ with thickness approximately $3 \mathrm{~nm}$. Therefore carriers are coherent all throughout these small geometries. Within a time scale shorter than the dephasing time $\stackrel{12}{=}$ the Rabi oscillation (RO) can provide a direct control of excited state population especially in strong excitation regime. It was proposed to be a good optical implement in quantum dot systems $\frac{13}{2}$ However, a simple two-level system involving Rabi oscillations with Rashba SOI in a coaxial double quantum ring has not yet been studied. Therefore, in this paper, we consider two-level and three-level models to explore spin-dependent electron dynamics assisted by $\mathrm{RO}$ processes. Under influences of the magnetic flux, the spin feature of the system is demonstrated only through effects of Rashba SOI. The presence of Rashba SOI also plays an important role in the mixture of neighboring angular momenta as well as spins that builds up a new selection rule and opens more dipole-allowed transition routes.

In view of quantum algorithm realization, the twolevel Rabi oscillators are often the prototype of the qubit generators. However, it is also important to establish coherent control in realistic multi-level quantum systems $\stackrel{14.15}{ }$ Hence, we explore the multi-level dynamical system involving the cascade-type and the $\Lambda$-type threelevel schemes driven by either sinusoidal impulsions or chirped laser pulses. To achieve efficient transfers, we employ adiabatic rapid passage method (ARP) $\stackrel{16,17}{,}$ namely that the excitation process rapid compared with the natural life time of an excited state in the limit of slowly varying detuning field, to simulate complete transfer processes. We will show that the probability of an electron that occupies the final state coincides with the estimation of the Landau-Zener formula in the adiabatic limit $\underline{18}$

The paper is organized as follows: In Sec. II the singleparticle Hamiltonian is derived and numerically solved and SOI accompanied AB energy spectrum is analyzed. In Sec. III ROs between two levels selected from the double-ring spectrum are studied. In Sec. IV photonassisted electron transitions in the three-level systems of the cascade- and $\Lambda$-schemes are investigated. Finally the 


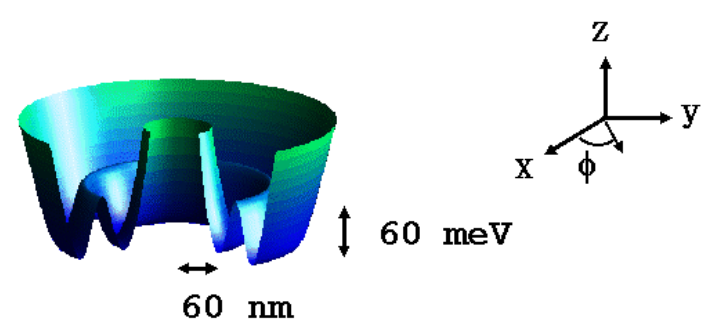

FIG. 1: (Color online) The diagram of part of the double-ring potential depicted in $0 \leq \phi \leq 3 \pi / 2$. The radius of the ring is about $160 \mathrm{~nm}$ and effective range of the magnetic flux $r_{\Phi}$ is about $7 \mathrm{~nm}$.

paper ends with a conclusion in Sec. V.

\section{THE ENERGY SPECTRA OF THE DOUBLE-RING SYSTEM}

The system of a double-ring 2DEG is enclosed by a magnetic flux in the presence of Rashba SOI. The electron is confined in an axial-symmetric potential $V_{c}$, shown in Fig. (10), which is exposed in a monochromatic electromagnetic (EM) field. In semiclassical description the Hamiltonian is given by

$$
\begin{aligned}
H= & \frac{1}{2 m^{*}}\left[\vec{p}-\frac{e}{c} \mathbf{A}(\vec{r}, t)\right]^{2}+V_{c}(r) \\
& +\frac{\alpha}{\hbar}\left[\vec{\sigma} \times\left(\vec{p}-\frac{e}{c} \mathbf{A}(\vec{r}, t)\right)\right]_{z}
\end{aligned}
$$

where $\mathbf{A}(\vec{r}, t)$ contains contributions from the magnetic flux and the EM field, and $\alpha$ is the coupling constant of Rashba SOI. The double-ring potential is modeled as

$$
V_{c}(r)=\frac{1}{2} m^{*} \omega_{0}^{2}\left(r-r_{0}\right)^{2}+\sum_{i=1}^{3} V_{i} e^{-\left(r-r_{i}\right)^{2} / \sigma_{i}^{2}},
$$

where $\omega_{0}$ is a factor defining the characteristic length $l_{c}=\sqrt{\hbar / m^{*} \omega_{0}}$ of the system and $\sigma_{i}$ is the Gaussian spatial width. The magnetic flux applied through the central region of the inner ring within $r_{\Phi}$ is described by the vector potential $\vec{A}_{\Phi}=B r / 2 \hat{\phi}$ for $r \leq r_{\Phi}$ and $\overrightarrow{A_{\Phi}}=B r_{\Phi}^{2} / 2 r \hat{\phi}$ for $r>r_{\Phi}$. Therein the unit vector in the angular direction $\hat{\phi}=-\sin (\phi) \hat{x}+\cos (\phi) \hat{y}$ has been used. The effect of the linearly polarized EM wave is simply expressed as $\overrightarrow{\mathcal{A}}_{E M}(t)=A_{0} \sin (k z-\Omega t) \hat{x}$, where $k$ and $\Omega$ are the wave vector and the frequency of the wave.

The Hamiltonian can be divided as $H=H_{0}+H_{\text {int }}$, where $H_{0}$ and $H_{\text {int }}$ correspond to the unperturbed and time-dependent Hamiltonian respectively. From energy conservation, the rapidly oscillating quadratic term in $\overrightarrow{\mathcal{A}}_{E M}(t)$ is omitted, and hence

$$
H_{\mathrm{int}} \simeq-\left\{\frac{e \overrightarrow{\mathcal{A}}_{E M}}{m^{*} c}\left(\vec{p}-\frac{e}{c} \vec{A}_{\Phi}\right)+\frac{\alpha e}{\hbar c}\left[\vec{\sigma} \times \overrightarrow{\mathcal{A}}_{E M}\right]_{z}\right\} .
$$

It is convenient to rewrite $H_{\mathrm{int}}=H_{D}+H_{B}+H_{S O}$ indicating three different types of interaction. The first term is the electron dipole interaction, given by

$$
H_{D}=\frac{-e}{m^{*} c} \overrightarrow{\mathcal{A}}_{E M} \cdot \vec{p} \simeq-e \vec{x} \cdot \overrightarrow{\mathcal{E}},
$$

where the time-dependent electric field, $\overrightarrow{\mathcal{E}}=\mathcal{E}_{0} \cos (\Omega t) \hat{x}$, is polarized along $\mathbf{x}$ direction. The second contribution $H_{B}$ is due to the applied magnetic flux

$$
\begin{aligned}
H_{B} & =\frac{e^{2}}{m^{*} c^{2}} \overrightarrow{\mathcal{A}}_{E M} \cdot \vec{A}_{\Phi} \\
& =\frac{e^{2} \mathcal{E}_{0}}{m^{*} c \Omega} A_{\Phi}(r) \sin (\phi) \sin (\Omega t) .
\end{aligned}
$$

The third term, $H_{S O}$, denotes the SO coupling mechanism

$$
\begin{aligned}
H_{S O} & =-\frac{\alpha e}{\hbar c}\left[\vec{\sigma} \times \overrightarrow{\mathcal{A}}_{E M}\right]_{z} \\
& =\frac{-\alpha e \mathcal{E}_{0}}{\hbar \Omega} \sigma_{y} \sin (\Omega t) .
\end{aligned}
$$

The electron dynamics can be derived based on the knowledge of eigenfunctions of $H_{0}$. At $t=0$ the normalized two-component wavefunction is $\Psi=\left(\Psi_{\uparrow}, \Psi_{\downarrow}\right)^{T}$, where

$$
\Psi_{\sigma}=\psi_{\sigma}(\vec{r}) \otimes \chi_{\sigma},
$$

with $\sigma=\uparrow$ or $\downarrow$ indicating two spin branches. Since total angular momentum $J_{z}$ commutes with time-independent Hamiltonian in the presence of SOI, the spatial wave function can be expressed of the form

$$
\begin{aligned}
& \psi_{\uparrow}(\vec{r})=\psi_{l_{\uparrow}}(r) e^{i l_{\uparrow} \phi} \\
& \psi_{\downarrow}(\vec{r})=\psi_{l_{\downarrow}}(r) e^{i l_{\downarrow} \phi},
\end{aligned}
$$

where the orbital angular momenta $l_{\uparrow}=m_{j}-1 / 2$ and $l_{\downarrow}=m_{j}+1 / 2$ follow the relation $l_{\downarrow}=l_{\uparrow}+1$, with $m_{j}$ corresponding to the eigenvalue of $J_{z}$. While dipole interaction does not flip spin directly the spin flipping is possibly achieved in the presence of the SOI and therefore we can investigate the spin-dependent charge dynamics. To characterize this feature, we define the net spin polarizability

$$
\mathcal{P}=\frac{\left\langle\Psi\left|\sigma_{z}\right| \Psi\right\rangle}{\langle\Psi \mid \Psi\rangle}=\frac{\left\langle\Psi_{\uparrow} \mid \Psi_{\uparrow}\right\rangle-\left\langle\Psi_{\downarrow} \mid \Psi_{\downarrow}\right\rangle}{\langle\Psi \mid \Psi\rangle} .
$$

For the case of $|\mathcal{P}|=1$, this indicates that the system is totally polarized into spin- $\uparrow($ spin- $-\downarrow)$ if $\mathcal{P}=+1(\mathcal{P}=$ -1 ). Otherwise, the spin polarizability can be generally specified by the notation $\mathcal{P}_{\uparrow}$ if $\mathcal{P}>0$, and $\mathcal{P}_{\downarrow}$ if $\mathcal{P}<0$.

Specifically, we consider an InAs-based double quantum ring system, of which the quantum structures are appropriate to investigate some spin-related phenomena. $\underline{19}$ Below we have selected the InAs material parameters $m^{*} / m_{0}=0.042$ and $\alpha \sim 40 \mathrm{meV} \cdot \mathrm{nm}$. Correspondingly, 
the characteristic energy $E=\hbar \omega_{0}=5 \mathrm{meV}$ and for the EM field $\hbar \Omega=1 \mathrm{meV}$. Dimensionless parameters of the double-ring potential are: $r_{c}=0, V_{1}=70, V_{2}=20$, $V_{3}=-20, \sigma_{1}=1.825, \sigma_{2}=1.0, \sigma_{3}=2.236, r_{1}=0$, $r_{2}=5.0$, and $r_{3}=6.0$. In the numerical calculation, we shall present the magnetic flux in units of the flux quantum $\Phi_{0}=h c / e$.

By choosing the above typical physical quantities and diagonalizing the time-independent Hamiltonian, it is easy to obtain the single-particle energy spectrum as is shown in Fig. (2a). As compared with the energy spectra in usual quantum dots, a genuine effect of SOI can be revealed in the reduction of degeneracies from fourfold to at most two-fold. But for rings there are only two-fold degeneracies at zero magnetic flux whether the SOI exists or not. The presence of the magnetic flux breaks the time reversal symmetry but Kramer's degeneracy is not lifted due to the absence of the Zeeman effect. In Fig. (2a), the solid curves indicate energy levels of positive $m_{j}$ whereas those of negative $m_{j}$ are depicted by dashed curves. The lowest five pairs of energy levels belong to $\left|m_{j}\right|=2.5,3.5,1.5,4.5$, and 0.5 . The ordering of these levels could change if the coupling constant $\alpha$ of the Rashba SOI is varied. For instance, when $\alpha=5 \mathrm{meV} \cdot \mathrm{nm}$, the lowest five pairs of these levels are with $\left|m_{j}\right|=0.5,1.5,0.5,2.5$, and 1.5. In the concern of varying $\alpha$, one can drive coherent ROs and the idea has been realized in quantum dot systems $\underline{\underline{20}}$

In the absence of the magnetic flux, the second and the third (as well as the fourth and the fifth, etc.) levels in Fig. (22) are close to each other. The gap between these adjacent levels arises from the zero-field splitting of the Rashba SOI, which will disappear when the SOI coupling constant $\alpha$ approaches zero. In the limit $\alpha \rightarrow 0$ not only the adjacent levels at $\Phi / \Phi_{0}=0$ mentioned above but also the curves split from these levels in the region $\Phi / \Phi_{0}>0$ will merge together. Another decisive feature distinguishing quantum rings from quantum dots stands out that for the former the ground state will periodically shift to that of higher total angular momentum. However, it always corresponds to the state with the lowest angular momentum in quantum dots.

An energy level $E$ in Fig. (2a) is a piecewise smooth function of $\Phi / \Phi_{0}$ with singular crossing points. The zigzag thick curve shows an example of the sixth lowest level, which has five crossing points around $\Phi / \Phi_{0}=$ $0.5,0.6,1.0,1.4$ and 1.5 within the unit interval $0.5<$ $\Phi / \Phi_{0}<1.5$ at $\alpha=40 \mathrm{meV} \cdot \mathrm{nm}$. If $\alpha \rightarrow 0$, the pairs of curves merge as discussed above and the set of crossing points reduce to $\Phi / \Phi_{0}=0.5$ and 1.5 for the ground state, and reduce to $\Phi / \Phi_{0}=0.5,1.0$, and 1.5 for other levels. It turns out that at $\alpha=0$ the electron in the double-ring reveals a similar oscillation pattern, with the same oscillation period one, as the $\mathrm{AB}$ oscillations in a single ring without SOI $\stackrel{21,22}{=}$ For $\alpha>0$, the splitting of local spin branches in $\mathrm{AB}$ oscillating spectrum of a single ring can be identified. ${ }^{23}$ For the double ring, spectral patterns become more complicated, but patterns with reg- ular oscillations are still rather apparent. Moreover, the spin polarizability $\mathcal{P}$ defined in Eq. (9) varies in $\Phi / \Phi_{0}$. It seems that the crossing points of the lowest level in Fig. (22), i.e., at $\Phi / \Phi_{0}=\{0.5,1,1.5,2, \ldots\}$ are exactly the positions where $\mathcal{P}$ changes its sign, at least in the range we studied.
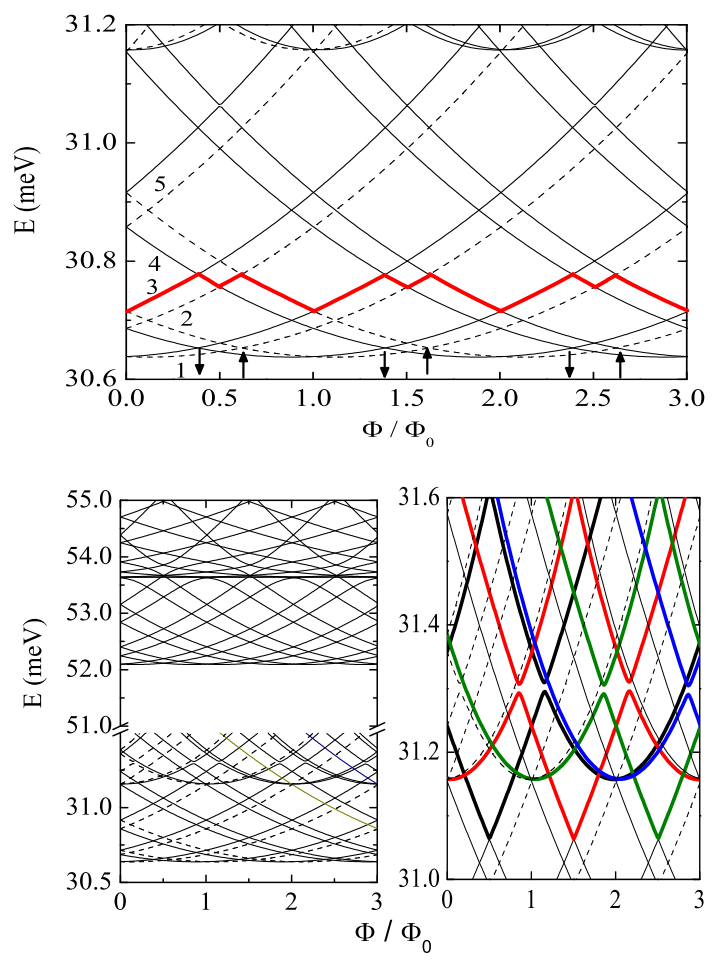

FIG. 2: (Color online) (a) The Aharonov-Bohm oscillations in the energy spectrum of the double ring in the presence of the Rashba SOI with $\alpha=40 \mathrm{meV} \cdot \mathrm{nm}$ and a static magnetic flux. The spectrum of states with positive (negative) $m_{j}$ are depicted in solid (dashed) curves. The lowest five pairs of the states are specified by $\left|m_{j}\right|=2.5,3.5,1.5,4.5$, and 0.5 . In each $1 / 2-\Phi / \Phi_{0}$ region, up or down arrows denote net spin orientations of ground states. The zigzag curve shows the sixth lowest eigenenergy. (b) Energy levels within lowest four subbands, in which energies of subband bottoms line close by $30.6,31.2,52.1$, and $53.6 \mathrm{meV}$, respectively. (c) Near the second subband bottom, anti-crossing levels are depicted in thick curves. From left to right, these states are of $m_{j}=0.5$ (black), 2.5 (green), 1.5 (red), and 3.5 (blue).

To explain the location of the crossing points in Fig. (2a), let us consider an ideal one-dimensional ring of radius $R$ enclosing a magnetic flux. The spectral property of this system reflects some key features of a radial subband of the double ring (Fig. (2b)). The fluxdependent energy spectrum can be derived in the analytical form 


$$
E=\frac{\hbar \omega_{a}}{2}\left\{\left(l_{\uparrow}-\frac{\Phi}{\Phi_{0}}\right)^{2}+\left(l_{\downarrow}-\frac{\Phi}{\Phi_{0}}\right)^{2} \pm \sqrt{\left.\left[\left(l_{\uparrow}-\frac{\Phi}{\Phi_{0}}\right)^{2}-\left(l_{\downarrow}-\frac{\Phi}{\Phi_{0}}\right)^{2}\right]^{2}+\frac{4 \alpha^{2}}{R^{2}} \frac{1}{\hbar^{2} \omega_{a}^{2}}\left(l_{\uparrow}-\frac{\Phi}{\Phi_{0}}\right)\left(l_{\downarrow}-\frac{\Phi}{\Phi_{0}}\right)\right\}}\right.
$$

where $\hbar \omega_{a}=\hbar^{2} / 2 m^{*} R^{2}$. Due to the relation $l_{\downarrow}=l_{\uparrow}+1$, the energy $E$ can be expressed as a function of the variables $l_{\downarrow}$ and $\Phi / \Phi_{0}$. A crossing point will come up at a certain $\Phi / \Phi_{0}$ where different integers $l_{\downarrow}$ have the same energy $E$. According to Eq. (10), this happens when $\Phi$ increases from zero to a period $\Phi_{0}$ if the Rashba SOI is absent. In the presence of the Rashba SOI, additional crossing points appear before $\Phi$ reaches $\Phi_{0}$. As compared to the Fock-Darwin spectrum in a quantum dot where the energy is in linear proportion to the trapping frequency and the cyclotron frequency in weak and strong magnetic fields respectively. However, while SOI can be regarded as the perturbation, Eq. (10) well tells a quadratic relation with the magnetic flux.

Level crossing points can be easily seen in Fig. (2a). Anti-crossing levels also appear, for instance in the example of Fig.(2r), and even in high energy regimes, as seen in the two dashed lines at $\left(\Phi / \Phi_{0}, E\right)=(1.7,53.7)$ in Fig. (4a). While the splitting of the accidental level degeneracy in quantum dots has been demonstrated both theoretically and experimentally, ${ }^{24}$ the repulsions in the avoiding levels due to the interplay between Zeeman and Rashba terms are also reported recently. ${ }^{25}$ In our case, anti-crossings near the second subband bottom arises in the presence of strong SOI. But for high energy pairs, the repulsions here are mainly attributed to the geometric effect of the double ring under influences of the magnetic flux. In other words, the repulsion levels in the double ring will not disappear, even when Rashba effect is turned off. In the vicinity of the minimal splitting points, wavefunctions vary acutely and cannot be specified by a set of good quantum numbers. The doublering Hamiltonian thus typically manifests the signature of quantum chaos. A comparison between the spectra of $\alpha=0$ and $20 \mathrm{meV} \cdot \mathrm{nm}$ shows that the Rashba SOI will increase the level splitting in each energy pair mentioned above but decrease the gap of the repulsion levels from 0.32 to $0.28 \mathrm{meV}$. Moreover, by adiabatically modulating the gate voltage to change SOI, the double-ring system discussed here serves as a candidate for testing the Berry phase. ${ }^{26}$

\section{RABI OSCILLATIONS IN TWO-LEVEL TRANSITIONS}

Since an electron in the inner (outer) ring has a definite angular momentum, its tunneling probability to the neighbor ring is suppressed under the constrain of the angular momentum conservation. Therefore, an eigen- function in the double ring may be localized only in the inner or the outer ring, if its corresponding energy is lower than the barrier. In the following we are going to investigate the dynamics of an electron under irradiations of an external EM field in the presence of SOI. The transition between two such kind of quantized levels in the energy space corresponds to an inter-ring transition in the spatial space.

We consider arbitrary two levels in the double ring. Suppose the electron initially occupies state $|b\rangle$ with eigenfunction $u_{b}(\vec{r})$ in the outer ring, the irradiation process is designed to pump it to state $|a\rangle$ with eigenfunction $u_{a}(\vec{r})$ in the inner ring. For convenience the timedependent wave function can be written as

$$
\psi(\vec{r}, t)=c_{a}(t) e^{i\left(\delta / 2-\omega_{a}\right) t} u_{a}(\vec{r})+c_{b}(t) e^{i\left(-\delta / 2-\omega_{b}\right) t} u_{b}(\vec{r}),
$$

where $u_{a}(\vec{r})$ and $u_{b}(\vec{r})$ associated with $E_{j}=\hbar \omega_{j}$ for $j=a$ and $b$, related to two-component wave function $\Psi$ in Eq. (7), as eigenfunctions and eigenenergies of $H_{0}$. Moreover, an optical transition takes place between two states that correspond to dipole-allowed eigenstates conforming to the relation $\Delta m_{j}= \pm 1$. As usual, $\delta \equiv(\Delta E / \hbar-\Omega)$ is the detuning defined as the frequency difference between the level spacing and the laser field as shown in the inset of Fig. (3a). Insert Eq. (11) into time-dependent Schrödinger equation, the time-evolution of an electron can be expressed as

$$
\left[\begin{array}{c}
\dot{c_{a}}(t) \\
\dot{c_{b}}(t)
\end{array}\right]=\frac{i}{2}\left[\begin{array}{cc}
-\delta & R_{D}+\tilde{R} \\
R_{D}^{*}+\tilde{R}^{*} & \delta
\end{array}\right]\left[\begin{array}{c}
c_{a}(t) \\
c_{b}(t)
\end{array}\right]
$$

with $\tilde{R}=R_{A}+R_{S O}$, which after some calculations have the relations

$$
\begin{aligned}
R_{D} & =e \mathcal{E}_{0}\left\langle u_{a}(r)|r| u_{b}(r)\right\rangle / 4 \hbar \\
R_{B} & =e^{2} \mathcal{E}_{0}\left\langle u_{a}(r)\left|A_{\Phi}(r)\right| u_{b}(r)\right\rangle / 4 \hbar m^{*} c \Omega, \\
R_{S O} & =\alpha e \mathcal{E}_{0}\left\langle u_{a}(r) \mid u_{b}(r)\right\rangle / 2 \hbar^{2} \Omega .
\end{aligned}
$$

Therein, $R_{D}$ is the dipole-induced Rabi frequency as usually discussed and $R_{B}$ and $R_{S O}$ denote the couplings of the laser field with the magnetic flux respectively the Rashba SOI. In calculating $R_{S O}$, only inner products between partial waves with different spin orientations would be taken into account. In deriving Eq. (12), we utilized the rotating-wave approximation (RWA) and ignored the counter-rotating terms proportional to $\exp [ \pm i(\Omega+\Delta E / \hbar) t]$.

For an electron initially occupies the low energy state $|b\rangle$ the time-dependent population probability can be ex- 
actly written as

$$
\begin{aligned}
& \left|c_{a}\right|^{2}=\frac{\tilde{R}_{\mathrm{eff}}^{2}}{\Omega_{d}^{2}} \sin ^{2}\left(\frac{\Omega_{d} t}{2}\right) \\
& \left|c_{b}\right|^{2}=1-\frac{\tilde{R}_{\mathrm{eff}}^{2}}{\Omega_{d}^{2}} \sin ^{2}\left(\frac{\Omega_{d} t}{2}\right),
\end{aligned}
$$

where $\Omega_{d}^{2}=\tilde{R}_{\text {eff }}^{2}+\delta^{2}$ and $\tilde{R}_{\text {eff }}=R_{D}+\tilde{R}$ can be regarded as the effective Rabi frequency in the presence of the external fields and the SOI. The on-resonance transitions occur when $\delta=0$, in the case the population probability can be simply reduced to $\left|c_{a}\right|^{2}=\sin ^{2}\left(\tilde{R}_{\text {eff }} t / 2\right)$ and $\left|c_{b}\right|^{2}=\cos ^{2}\left(\tilde{R}_{\mathrm{eff}} t / 2\right)$.

If the energy dissipation to the environment is considered as the interaction between an electron with continuous vacuum modes, a phenomenological decay parameter $\gamma$ will be introduced to the first element of the matrix in Eq. (12), which opens a decay path from the excited state to its surrounding. Thus the coefficients describing the system will be changed in the more complicated form

$$
\begin{aligned}
& \left|c_{a}\right|^{2}=\frac{\tilde{R}_{\text {eff }}^{2}}{\tilde{\Omega}^{2}} e^{-\gamma t} e^{-\tilde{\Omega} \zeta t} \sin ^{2}\left(\frac{\tilde{\Omega} \eta t}{2}\right) \\
& \left|c_{b}\right|^{2}=\frac{e^{-\gamma t}}{\tilde{\Omega}^{2}} e^{-\tilde{\Omega} \zeta t}\left[\left(\gamma^{2}+\delta^{2}\right) \sin ^{2}\left(\frac{\tilde{\Omega} \eta t}{2}\right)+\tilde{\Omega}^{2} \cos ^{2}\left(\frac{\tilde{\Omega} \eta t}{2}\right)+2 \tilde{\Omega}(\gamma \eta-|\delta| \zeta) \sin (\tilde{\Omega} \eta t)\right],
\end{aligned}
$$

where $\zeta=\cos \left(\theta_{1} / 2\right)$ and $\eta=\sin \left(\theta_{2} / 2\right)$, with

$$
\begin{aligned}
\theta_{1} & =\cos ^{-1}\left[\left(\gamma^{2}-\Omega_{d}^{2}\right) / \tilde{\Omega}^{2}\right] \\
\theta_{2} & =\sin ^{-1}\left[2|\delta| \gamma / \tilde{\Omega}^{2}\right] \\
\tilde{\Omega}^{2} & =\sqrt{\left(\gamma^{2}-\Omega_{d}^{2}\right)^{2}+(2 \delta \gamma)^{2}},
\end{aligned}
$$

in which $\theta_{1}$ and $\theta_{2}$ should be taken from the same quadrant.

For convenience, we introduce the notation $\left|m_{j}\right\rangle_{\mathcal{P}}$ to specify a state in terms of the total angular momentum $m_{j}$ and its spin polarizability $\mathcal{P}$. We choose two states $|a\rangle=|-2.5\rangle_{\mathcal{P}_{\uparrow}}$ and $|b\rangle=|-1.5\rangle_{\mathcal{P}_{\downarrow}}$ respectively, at $\Phi=1.3 \Phi_{0}$ with $E_{a}=37.03 \mathrm{meV}$ and $E_{b}=30.64 \mathrm{meV}$, as depicted in Fig. (3a). The corresponding population inversion

$$
W(t)=\left|c_{b}(t)\right|^{2}-\left|c_{a}(t)\right|^{2}
$$

of this system is demonstrated in Fig. 3(b). If the vacuum fluctuation is absent $(\gamma=0)$, the total probability is conserved, i.e., $\left|c_{b}(t)\right|^{2}+\left|c_{a}(t)\right|^{2}=1$ at any time. In this case $W(t)$ manifests oscillating behavior within the interval $\left[1-2\left(\tilde{R}_{\mathrm{eff}}^{2} / \Omega_{d}^{2}\right), 1\right]$ without any dissipation. For $\delta \rightarrow 0$, this interval approaches to its maximum values $[-1,1]$, namely that $\Omega_{d} \rightarrow \tilde{R}_{\text {eff }}$. For large detuning, e.g. $\delta=0.2$, this interval will shrink to its $70 \%$, and the blue shifted inversion curve is plotted in the dotted line in Fig. (3b).

If the spontaneous emission of an excited state is considered, $W(t)$ will decay with time, as shown by the solid curve in Fig. (3) ), in which $\gamma=0.03$ and $\delta=0.02$. In this case $W(t)$ oscillates underdamped. The damping behavior which in consistent with the Weisskopf-Wigner theory ${ }^{27}$ is well-fitted by $W_{\text {fit }}=e^{-\Gamma t}$, where the Fermi's rate $\Gamma \sim 0.03$. When the Rabi relaxation time is defined as $\tau_{R}=1 / \Gamma$, our calculation shows that a cycle of inter-ring transition accomplishes in $\tau_{R}$ for a given $\gamma$. While the spontaneous decay can be experimentally controlled and suppressed,, 28 in a cavity with a limited number of modes at the transition frequency, a long decoherence time is permitted. In assumption of weak systemenvironment coupling, efficient population transfers are feasible. So in conclusion, under SOI we can simultaneously manipulate electron transitions associated with its spin orientations in either rings via the RO processes of a two-level model.

\section{THE PHOTON-ASSISTED TRANSITIONS IN THREE-LEVEL SCHEMES}

In this section we apply the Rabi model to several interesting cases. We show how inter-ring transition are achieved via the photon-assisted processes. The processes are demonstrated by considering threelevel systems in cascade-type and $\Lambda$-type schemes that are shown in Fig. (4b) and Fig. (5b). For clarity, we rewrite the time-dependent wave function as $\psi(\vec{r}, t)=$ $\sum_{j=1}^{3} c_{j}(t) e^{-i \omega_{j} t} u_{j}(\vec{r})$, where $E_{j}=\hbar \omega_{j}$ and we define $E_{12}=E_{2}-E_{1}, E_{13}=E_{3}-E_{1}, E_{12}+\delta=E_{13}-\delta=\hbar \Omega$. The involved states construct two dipole-allowed transitions $\{|1\rangle \leftrightarrow|2\rangle\},\{|1\rangle \leftrightarrow|3\rangle\}$ and a $\{|2\rangle \leftrightarrow|3\rangle\}$ dipoleforbidden paths. For a clear demonstration we shall ignore spontaneous emission processes.

\section{A. The cascade-type}

It is well known that the quantum-beat spectroscopic method permits the resolution of closely neighboring levels. ${ }^{29}$ Earlier experiments demonstrate that quantum- 


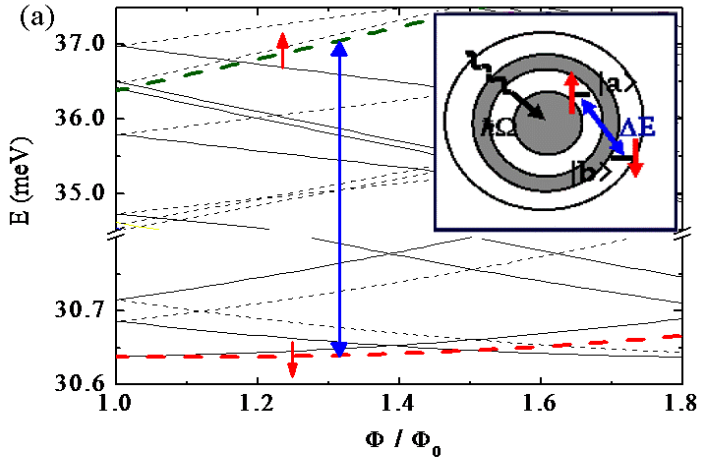

(b)

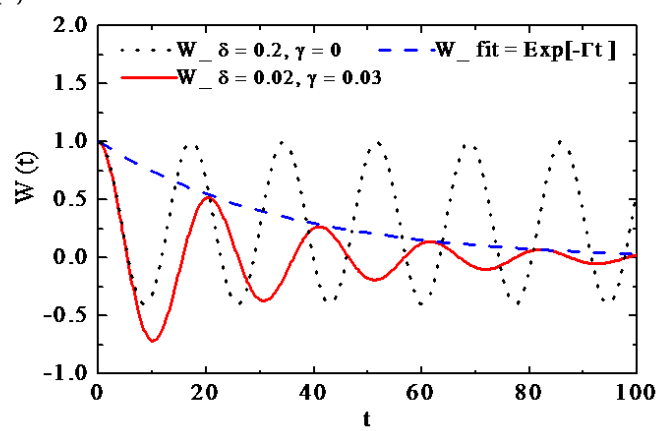

FIG. 3: (Color online) (a) The energy spectrum including two states: $|a\rangle=|-2.5\rangle_{\mathcal{P}_{\uparrow}}$ and $|b\rangle=|-1.5\rangle_{\mathcal{P}_{\downarrow}}$. The sketch in the inset depicts inter-ring transitions under EM wave stimulations. (b) Population inversion as the function of time. When ignoring the spontaneous emission, $W(t)$ with large detuning is demonstrated by dotted symbol. If the spontaneous emission of an excited state is considered, then in small detuning regime, where we set $\gamma=0.03$ and $\delta=0.02, W(t)$ manifests an underdamped oscillation as depicted by the solid curve and the decay behavior is fitted by an exponential decay function as is shown by the dashed line.

beat spectroscopy is a useful technique in the measurement of Zeeman splittings and hyperfine intervals in

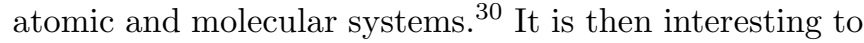
study spectroscopic dynamics involving the direct interring transitions in the cascade-type scheme nearby the avoided crossing points.

Again, we use the notation $\left|m_{j}\right\rangle_{\mathcal{P}}$ to specify the involving states. Here we consider an up-spin state $|1\rangle=$ $|-1.5\rangle_{\mathcal{P}_{\uparrow}}$, and a down-spin doublet $|2\rangle=|-2.5\rangle_{\mathcal{P}_{\downarrow}}$ and $|3\rangle=|-2.5\rangle_{\mathcal{P}_{\downarrow}}$ at $\Phi=1.7 \Phi_{0}$. For state $|1\rangle$, the electron is localized in the inner ring. The electron wave functions, however, extend over the double ring for two higher states. The energy spectrum is depicted in Fig. (4h), and in (b) we show the sketch of the cascade model. Similar to solving Eq. (12) for the two-level system, transitions among different spin states can be investigated through

$$
\begin{aligned}
\dot{c_{1}}(t) & =\frac{i}{2}\left[e^{-i \delta t} R_{+}^{12} c_{2}(t)+e^{i \delta t} R_{-}^{13} c_{3}(t)\right] \\
\dot{c_{2}}(t) & =\frac{i}{2} e^{i \delta t} R_{+}^{* 21} c_{1}(t) \\
\dot{c_{3}}(t) & =\frac{i}{2} e^{-i \delta t} R_{-}^{* 31} c_{1}(t),
\end{aligned}
$$

where $R_{ \pm}^{i j}=R_{D}^{i j} \pm \tilde{R}^{i j}$. Adopting transformations $c_{2}(t)=C_{2}(t) e^{i \delta t}$ and $c_{3}(t)=C_{3}(t) e^{-i \delta t}$, Eq. (16) can be reexpressed as a homogeneous autonomous equation. For an electron initially occupies state $|1\rangle$, the population probability in small detuning regime can be expressed approximately to

$$
\begin{aligned}
\left|c_{1}(t)\right|^{2} & =\cos ^{2}\left(\frac{M_{R} t}{2}\right) \\
\left|c_{2}(t)\right|^{2} & =\left(\frac{R_{+}^{12}}{M_{R}}\right)^{2} \sin ^{2}\left(\frac{M_{R} t}{2}\right) \\
\left|c_{3}(t)\right|^{2} & =\left(\frac{R_{-}^{13}}{M_{R}}\right)^{2} \sin ^{2}\left(\frac{M_{R} t}{2}\right),
\end{aligned}
$$

where $M_{R}=\sqrt{\left(R_{+}^{12}\right)^{2}+\left(R_{-}^{13}\right)^{2}}$. Beyond the small detuning approximation, Eq. (16) is numerically solved and

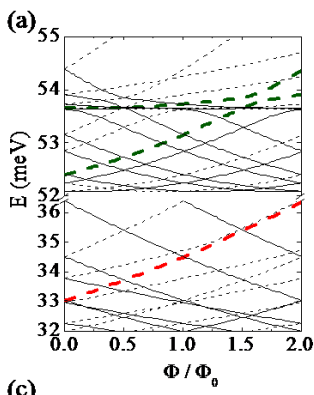

(b)

(c)

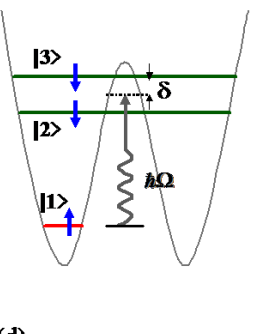

(d)

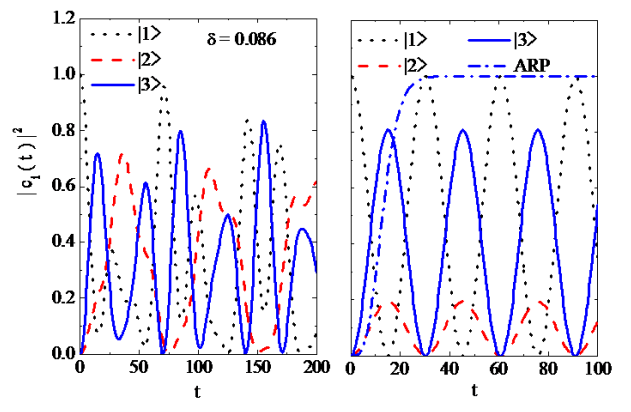

FIG. 4: (Color online) (a) The energy spectrum including the chosen three levels, an up-spin state $|1\rangle=|-1.5\rangle_{\mathcal{P}_{\uparrow}}$ (red), and a down-spin doublet $|2\rangle=|-2.5\rangle_{\mathcal{P}_{\downarrow}}$ and $|3\rangle=|-2.5\rangle_{\mathcal{P}_{\downarrow}}$ (green) at $\Phi=1.7 \Phi_{0}$. Level repulsion occurs between the doublet, and the gap is about $0.17 \mathrm{meV}$ for $\alpha=40 \mathrm{meV} \cdot \mathrm{nm}$. (b) A sketch of a cascade-type model in the double ring. population as a function of time for the cases of (c) $\delta=0.086$ and $(\mathrm{d}) \delta \rightarrow 0$. Dash-dotted curve in (d) shows the ARP estimation of the transition from $|2\rangle$ to $|3\rangle$. 
the result for $\delta=0.086$ is shown in Fig. (4c). Since transition probabilities come up differently between two paths, namely $R_{+}^{12} \neq R_{-}^{13}$, occupations with time on each states turn into be aperiodic and less regular compared with probabilities obtained from Eq. (17). Discrepancies between Fig. (4k) and Fig. (4d) are clearly illustrated. However, in the case $\delta \rightarrow 0$, the electron tends to oscillate between $|3\rangle$ and $|1\rangle$ and the transition $|2\rangle \leftrightarrow|3\rangle$ is less efficient, as compared with the off-resonance transitions.

This drawback can be removed by another interesting manipulation in the cascade scheme, namely, by transferring electrons ladder by ladder with chirped laser pulses. The idea originates from the electron transfer in molecules, in which stepwise excitations are applied for a rapid and efficient dissociation of specific chemical bonds. ${ }^{31}$ Under ARP condition, electrons that are resonantly pumped to state $|2\rangle$ could be efficiently transited to the final state $|3\rangle$ in a long trapping time.

To this end, we select a chirped laser pulse with timedependent electric field along the radial direction, given by

$$
\overrightarrow{\mathcal{E}}_{c h}(t)=\mathcal{E}_{c h} \exp \left(-\frac{t^{2}}{2 \tau^{2}}-i \Omega_{c h} t-i \beta \frac{t^{2}}{2}\right) \hat{r},
$$

where $\tau$ is the pulse duration, $\Omega_{c h}$ is the central frequency, and $\beta$ is the temporal chirp. The equation of motion in RWA modified from Eq. (12) becomes

$$
\left[\begin{array}{c}
\dot{c_{2}}(t) \\
\dot{c_{3}}(t)
\end{array}\right]=\frac{i}{2}\left[\begin{array}{cc}
0 & R_{c h}(t) \\
R_{c h}^{*}(t) & 2 \delta_{c h}(t)
\end{array}\right]\left[\begin{array}{l}
c_{2}(t) \\
c_{3}(t)
\end{array}\right],
$$

in which $\delta_{c h}(t)=\beta t$ is linearly chirped detuning, and $R_{c h}(t)$ stands for the time-dependent Rabi frequency related the pulse envelope of $\overrightarrow{\mathcal{E}}_{c h}(t)$. By choosing proper parameters for a pulse with peak Rabi frequency $R_{0}=0.25$, $\beta=0.01$, and $\tau=10$, a complete transfer is demonstrated by the dash-dotted line in Fig. 4(d)). The numerical result coincides with the estimation of the LandauZener formula 18

$$
P \simeq 1-\exp \left(-\pi \frac{R_{0}^{2}}{2 \beta}\right) .
$$

In the adiabatic limit $|\beta| \ll R_{0}^{2}$, electrons have great probability to occupy an excited state in the long time limit. Distinguished time-evolution populations between stimulated transitions by CWs and steady transfer by a chirp pulse are depicted by curves in Fig. (4d). Therefore, in addition to the manipulation of electron transitions between a single ring and a double ring via the subjection to CW irradiations, we also arrive at the optimal control on stably selective excitations with chirped pulses in cascade-type systems.

\section{B. The $\Lambda$-type transition}

Finally we shall also investigate an interesting and important phenomenon: Whenever there are level cross- ings for two energy states that belong to either one ring, $\Lambda$-type scheme of indirect inter-ring transitions among which and one higher energy state can be switched on. Applications of this model has been proposed both in superconducting quantum interference device (SQUID) ${ }^{33}$ and semiconductor double quantum dots,, 34 in which multi-level ROs as a target towards coherent control has been demonstrated.

As Fig. (5a) shows that for an electron occupies state $|2\rangle$ the photon-assisted quantum transition is initiated from the inter-ring surpassing an intermediate-state $|1\rangle$ and finally reach to the outer-ring of state $|3\rangle$. Onresonance solutions of the mediated-indirect-transition system with initial conditions $c_{1}(0)=0, c_{2}(0)=1$, and $c_{3}(0)=0$ are

$$
\begin{aligned}
\left|c_{1}(t)\right|^{2}= & \left(\frac{R_{+}^{12}}{M_{R}}\right)^{2} \sin ^{2}\left(\frac{M_{R} t}{2}\right) \\
\left|c_{2}(t)\right|^{2}= & \left(\frac{R_{+}^{12}}{M_{R}}\right)^{4} \cos ^{2}\left(\frac{M_{R} t}{2}\right)+\left(\frac{R_{-}^{13}}{M_{R}}\right)^{4} \\
& +\frac{2\left(R_{+}^{12} R_{-}^{13}\right)^{2}}{M_{R}^{4}} \cos \left(\frac{M_{R} t}{2}\right) \\
\left|c_{3}(t)\right|^{2}= & \frac{\left(R_{+}^{12} R_{-}^{13}\right)^{2}}{M_{R}^{4}}\left[\cos \left(\frac{M_{R} t}{2}\right)-1\right]^{2}
\end{aligned}
$$

and the transition probabilities are shown in Fig. (5]). The incomplete transfer and occupation are restricted due to unequal effective Rabi frequencies in two paths. Once the external field optimizes the efficiency of one path, the efficiency of the other is not optimal.

The formulae in Eq. (21) clearly show that the probability $\left|c_{3}(t)\right|^{2}$ has a maximum value at $t=m \pi / M_{R}$ with an odd integer $m$ and a minimum value at $t=n \pi / M_{R}$ with an even integer $n$. At these extreme points we have the ratio $\left|c_{2}(t)\right|^{2} /\left|c_{3}(t)\right|^{2}=$ $\left[\left(R_{+}^{12}\right)^{2}-\left(R_{-}^{13}\right)^{2}\right]^{2} / 4\left(R_{+}^{12}\right)^{2}\left(R_{-}^{13}\right)^{2}$, which indicates the optimal transfer at $\left|R_{+}^{12}\right| /\left|R_{-}^{13}\right|=1$. Away from this ratio, the transfer efficiency will decrease. In Fig. (5] b), transition among a doublet of $m_{j}=0.5$ and 2.5 and an auxiliary level of $m_{j}=1.5$ at $\Phi=0.8 \Phi_{0}$ is considered. In this case, there is only $45 \%$ occupation on $|3\rangle$ for $R_{+}^{12} / R_{-}^{13} \sim 0.38$. If the effective Rabi frequencies of the paths $2 \leftrightarrow 1$ and $1 \leftrightarrow 3$ are the same, the optimal control of electron dynamics is feasible. To show this, we calculate the time-dependent occupation probability on each states. In the inset, it is clear that at extreme times, occupation on $|3\rangle$ based on a pseudo $\Lambda$ - transition process has a maximum value. Meanwhile, states $|1\rangle$ and $|2\rangle$ are empty left. Moreover, we also investigate the transition process stimulated by chirped pulse irradiation. By setting $R_{0}=0.25, \beta=0.001$, and $\tau=14.15$, we obtain a long-time occupation of the final state, obeying estimation of Landau-Zener's formula. The result is shown by dash-dotted line in the inset.

Within proper controls of the pulse width $\tau$ against the typical level spacings and the dephasing time of electrons, 

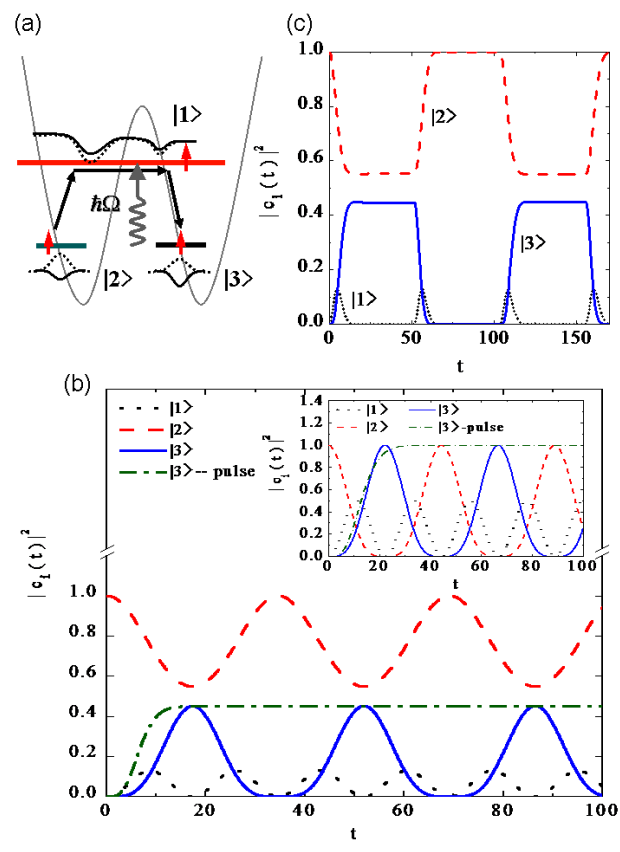

FIG. 5: (Color online) (a) A sketch of the $\Lambda$-type scheme. States here are all with effective up-spin orientations. An electron initially occupies state $|2\rangle$ can be optically pumped to an outer-ring state $|3\rangle$ mediated by state $|1\rangle$. (b) The population probabilities among three levels. The long-time occupation of an excited state is feasible by applying an short and intense pulse. In the inset we show the optimal transfer is feasible provided that there is common Rabi frequency in the two paths. (c) An alternative output signal can be obtained under successive pulse stimulations.

it is easy to manipulate electronic states in $\Lambda$-scheme system by successive application of pulses $\frac{32}{2}$ Using the same parameters as in Fig. (5b), we simulate the pulseinduced periodic oscillations. Here the Gaussian pulse duration $\tau=7.3$ and the pulse interval is about $7 \tau$. Different from the sinusoidal ROs, an alternative square wave is shown in Fig. (55). Apparently, the level occupation time in both inner and outer ring is prolonged. Moreover, since the duration of the pulse is properly tuned, it can be expected that time evolution of the occupation should be complete in the pseudo $\Lambda$ - transition process. Otherwise, under-excitation or over-excitation takes place corresponding to the duration being too short or too long, respectively. Level occupation will never be or just be transiently complete. The well control of pulse delay time also shows the flexibility of manipulations on quantum states.

\section{CONCLUSION}

In this work we have studied magnetic-optical transitions in a semiconductor double ring in the presence of Rashba spin-orbit coupling and the magnetic flux. First, based on accurate numerical calculations we obtain
SOI-accompanied $\mathrm{AB}$ energy spectra and corresponding eigenstates. The presence of the SOI has important influences on the occurrence of level crossings showing the evidence both for the periodic orbital motion and spin flips. In addition, there are anti-crossing levels playing the role of the magnetic-resonant extraditions of electrons between inner and outer rings. In high energy regime, occurrence of avoided crossings indicates a chaotic signature in its classical analogy. To facilitate the peculiar features of the double-ring system we have designed some interesting dynamic processes such that the system can be easily explored experimentally in the near future.

We have studied the temporal evolution processes in a two-level and two three-level models. The interaction between external fields and the electron results in the successive stimulating absorption and emission of a photon and turn out as the effective Rabi oscillators. In the two-level model, we demonstrate an alternative manipulation of electrons transiting between two rings. In cascade scheme, aperiodic and incomplete population transfer is revealed under the sinusoidal field excitations. By appropriate tuning SOI strength, the gap between avoided crossing levels can be reduced such that the rectified output signals are measurable. Moreover, by short pulse excitations we also demonstrate the possibility on optimal control of selective and direct signals. In this work, only one lader transition is demonstrated, which however can be extended to multi-ladder transitions following the same principle. Finally, we have explored the photon-assisted tunneling in the $\Lambda$-type model. In addition to generation of ROs also we give the criterion of the most efficient transfer via the mediated-indirecttunneling paths. Further, by successive pulse irradiations the well control on pulse delay results in the time prolongation on state populations. We should also emphasize that in the $\Lambda$-type scheme the minimization of the intermediate-level population is achieved which is an important and practical strategy in device realization.

The presence of SOI allows the manipulation of spin degree of freedom and it is timely to examine the spindependent optical response. Since, similar features could be found in double-dot systems, the above theoretical results in a double ring might shed light on the future experimental findings in these burgeoning quantum systems. While there are few works on optically induced and SOI driven spin dynamics in quantum systems, $\stackrel{20,35,36}{\longleftarrow}$ we believe that the theoretical and experimental works of related spin read-out information by optical pumping in ring-like systems could be carried out in the near future.

\section{Acknowledgments}

This work was supported by National Science Council and Academia Sinica in Taiwan. The authors are grateful to valuable discussions with V. Gudmundsson, Y. N. Chen, G. Y. Chen, and W. Xu. 
1 P. A. Maksym and T. Chakraborty, Phys. Rev. Lett. 65, 108 (1990); T. Chakraborty, Comments Condens. Matter Phys. 16, 35 (1992); T. Chakraborty, Quantum Dots (North-Holland, Amsterdam, 1999); I. Magnusdottir and V. Gudmundsson, Phys. Rev. B 60, 16591 (1999); R. Krahne, V. Gudmundsson, C. Heyn1, and D. Heitmann1, ibid. 63, 195303 (2001).

2 J. Gorman, E. G. Emiroglu, and D. A. Williams, Phys. Rev. Lett. 95, 090502 (2005); T. Hayashi, T. Fujisawa, H. D. Cheong, Y. H. Jeong, and Y, Hirayama, Phys. Rev. Lett. 91, 226804 (2003); X. Li, Y. Wu, D. Steel, D. Garmon, T. H. Stievater, D. S. Katzer, D. Park, C. Oiermarocchi and L. J. Sham, Science 301, 809 (2003).

3 B. E. Kane, Nature (London) 393, 133 (1998); M. Friesen, C. Tahan, R. Joynt, and M. Eriksson, Phys. Rev. Lett.

4 L. P. Levy, G. Dolan, J. Dunsmuir, and H. Bouchiat, Phys. Rev. Lett. 64, 2074 (1990).

${ }^{5}$ V. Chandrasekhar, R. A. Webb, M. J. Brady, M. B. Ketchen, W. J. Gallagher, A. Kleinsasser, Phys. Rev. Lett. 67, 3578 (1991).

92, 037901 (2004); E. Paspalakis, Z. Kis, E. Voutsinas, and A. F. Terzis, Phys. Rev. B 69, 155316 (2004).

6 I. Zutic, J. Fabian, and S. Das Sarma, Rev. Mod. Phys. 76, 323 (2004).

7 B. Datta and S. Das, Appl. Phys. Lett 56, 665 (1990).

8 X. F. Wang, P. Vasilopoulos, and F. M. Peeters, Phys. Rev. B 65, 165217 (2002).

9 T. Koga, J. Nitta, H. Takayanagi, and S. Datta, Phys. Rev. Lett 88, 126601 (2002).

${ }^{10}$ W. H. Kuan, C. S. Tang, and W. Xu, J. Appl. Phys 95, 6368 (2004); O. Voskoboynikov, C. P. Lee, and O. Tretyak, Phys. Rev. B 63, 165306 (2001); M. V.-Rodriguez, A. Puente, and S. Serra, Phys. Rev. B 69, 085306 (2004); S. Debald and C. Emary, Phys. Rev. Lett. 94, 226803 (2005).

11 T. Mano, T. Kuroda, S. Sanguinetti, T. Ochiai, T. Tateno, J. Kim, T. Noda, M. Kawabe, K. Sakoda, G. Kido, and N. Koguchi , Nano Lett. 5, 425 (2005).

12 L. Allen and J. H. Eberly, Optical Resonance and TwoLevel Atoms (Dover Publications, Inc., New York, 1987).

${ }^{13}$ H. Kamada, H. Gotoh, J. Temmyo, T. Takagahara, and H. Ando, Phys. Rev. Lett. 87, 246401 (2001); T. H. Stievater, Xiaoqin Li, D. G. Steel, D. Gammon, D. S. Katzer, D. Park, C. Piermarocchi, and L. J. Sham, Phys. Rev. Lett. 87, 133603 (2001); H. Htoon, T. Takagahara, D. Kulic, O. Baklenov, A. L. Holmes, Jr., and C. K. Shih, Phys. Rev. Lett. 88, 087401 (2002); P. Borri, W. Langbein, S. Schneider, U. Woggon, R. L. Sellin, D. Ouyang, and D. Bimberg, Phys. Rev. B 66, 081306(R) (2002); S. Stufler, P. Ester, A. Zrenner, and M. Bichler, Phys. Rev. B 72, 121301(R) (2005).

14 W. S. Warren, H. Rabitz and M. Dahleh, Science 259, 1581 (1993); J. L. Krause and R. M. Whitnell, K. R. Wilson, and Y. J. Yan, in Femtosecond Chemistry, edited by J. Manz, L. Wöste (VCH, Weinheim, 1995), pp. 743-779; S. A. Rice M. Zhao, Optical Control of Molecular Dynamics (John Wiley \& Sons Inc., New York, NY, 2000).

15 S. Chelkowski, A. Bandrauk, and P. B. Corkum, Phys. Rev. Lett. 65, 2355 (1990); J. S. Melinger, D. McMorrow, C. Hillegas, and W. S. Warren, Phys. Rev. A 51, 3366
(1995); M. N. Kobrak, S. A. Rice, Phys. Rev. A 57, 2885 (1998).

16 J. S. Melinger, S. R. Gandhi, A. Hariharan, D. Goswami, and W. S. Warren, J. Chem. Phys. 101, 6439 (1994); V. S. Malinovsky and J. L. Krause, Eur. Phys. J. D 14, 147 (2001).

17 C. W. Hillegas, J. X. Tull, D. Goswami, D. Strickland, and W. S. Warren, Opt. Lett. 19, 737 (1994); M. M. Wefers, K. A. Nelson, Opt. Lett. 18, 2032 (1993).

18 L. D. Landau, Phys. Z. Sowj. 2, 46 (1932); C. Zener, Proc. R. Soc. Lond. A 137, 696 (1932).

19 D.Grundler, Phys. Rev. Lett. 84, 6074 (2000); C.-M. Hu, J. Nitta, T. Akazaki, H. Takayanagi, J. Osaka, P. Pfeffer, and W. Zawadzki, Physica E 6, 767 (2000).

20 S. Debald and C. Emary, Phys. Rev. Lett. 94, 226803 (2005).

21 A. Führer, S. Luscher, T. Ihn, T. Henzel, K. Ensslin, W. Wegsheider, and M. Bichler, Nature 413, 822 (2001); A. Lorke, R. J. Luyken, A. O. Govorov, J. P. Kotthaus, J. M. Garcia, and P. M. Petroff, Phys. Rev. Lett. 84, 2223 (2000).

22 T. Chakraborty and P. Pietiläinen, Phys. Rev. B 50, 8460 (1994); P. Pietiläinen and T. Chakraborty, Phys. Rev. B 73, 155315 (2006)

23 J. Splettstoesser, M. Governale, and U. Zülicke, Phys. Rev. B 68, 165341 (2003).

24 B. Jouault, G. Santoro and A. Tagliacozzo, Phys. Rev. B 61, 10242 (2000); L. P. Kouwenhoven, T. H. Oosterkamp, M. W. S. Danoesastro, M. Eto, D. G. Austing, T. Honda, and S. Tarucha, Science 278, 1788 (1997).

25 T. Chakraborty and P. Pietiläinen, Phys. Rev. Lett. 95, 136603 (2005).

26 D. Giuliano, P. Sodano, A. Tagliacozzo, Phys. Rev. B 67, 155317 (2003); S.-R. Eric Yang and N. Y. Hwang, Phys.Rev. B 73, 125330(2006).

27 In Methods in Theoretical Quantum Optics, edited by S. Barnett and P. Radmore (Clarendon Press, Oxford, 1997), Chapter 5.

${ }^{28}$ E. M. Purcell, Phys. Rev. 69, 681 (1946); E. Yablonovitch, Phys. Rev. Lett. 58, 2059 (1987); H. Hirayama, T. Hamano, and Y. Aoyagi, Appl. Phys. Lett. 69, 791 (1996).

${ }^{29}$ In The Physics of Atoms and Quanta, edited by H. Haken and H. Wolf (Springer, 1996), pp. 386-388.

30 S. Haroche, M. Gross, and M. P. Silverman, Phys. Rev. Lett. 28, 1063 (1974); R. Wallenstein, J. A. Paisner, and A. L. Schawlow, Phys. Rev. Lett. 32, 1333 (1974).

31 S. Chelkowski and G. Gibson, Phys. Rev. A 52, R3417 (1995); D. J. Mass, C. W. Rella, P. Antoine, E. S. Toma, and L. D. Noordam, Phys. Rev. A 59, 1374 (1999).

32 R. Gómez-Abal and W. Hübner, Phys. Rev. B 65, 195114 (2002).

33 Z. Zhou, S. I. Chu, and S. Han, Phys. Rev. B 66, 054527 (2002).

34 A. V. Tsukanov, Phys. Rev. B 73, 085308 (2006).

35 F. T. Vasko, Phys. Rev. B 70, 073305 (2004); A. Smirnov and L. Mourokh, Phys. Rev. B 71, 161305(R) (2005).

36 F. Malet, M. Pi, M. Barranco, E. Lipparini, and Ll. Serra, Phys. Rev. B 74, 193309 (2006). 